\title{
Testbed for Performance Evaluation of SAT-AIS Receivers
}

\author{
Andis Dembovskis \\ German Aerospace Center (DLR) \\ Institute of Space Systems \\ Bremen, Germany \\ Andis.Dembovskis@dlr.de
}

\begin{abstract}
The SAT-AIS technology, which offers global ship traffic monitoring from low earth orbit, is steadily gaining more attention within groups of communication hardware designers, as well as among safety policy chairman. Numerous research and business groups are elaborating hardware solutions. Motivated by testing needs as a result of the upcoming DLR AIS satellitemission AISat, a dedicated AIS Testbed for Satellite Systems was developed. This paper describes the approaches and procedures, which were identified as the most significant for testing SAT-AIS hardware.
\end{abstract}

Keywords-testbed; SAT-AIS; space; performance; evaluation

\section{INTRODUCTION}

AIS, which stands for Automatic Identification System, is a ship safety system imposed by the IMO (International Maritime Organization). It enables ships to monitor the surrounding traffic. Initially it was designed for terrestrial applications with 40 nautical miles visibility range, based on the SOTDMA (Self Organized TDMA) communication protocol [1]. However, within the last years a dedicated interest has arisen for ship monitoring from space, named "SAT-AIS", to derive surveillance of ship traffic around the globe. Several space AIS receivers have already been launched, proving the ability to receive AIS messages from LEO (Low Earth Orbit). In this context also the European Space Agency is working on a SATAIS satellite constellation setup for continuous and global ship monitoring in the frame of the ARTES 21 programme [8].

However there remains an important technological communication issue to be considered: since the AIS protocol was initially designed for terrestrial applications, there are significant signal distortions present for space application. These include low signal levels, packet overlapping and delays, signal Doppler shifts and phase distortions, which all have to be accounted for during the design and development of a high performance SAT-AIS receiver [2]. To assess how to best overcome the issue, a testbed allows the comparison of available receiver solutions for the targeted satellite mission.

The research and development of an AIS Testbed at the DLR Institute of Space Systems was initiated and motivated by the testing needs of own nanosatellite, AISat, due to carry different SAT-AIS payloads. This research has resulted in the solutions presented in this paper: the testbed is capable of de- riving receiver performance indicators using special quasi static test-scenarios, as well as simulated results for real-world ship traffic data scenarios for in-orbit operation.

\section{TESTBED DESIGN}

Fig. 1 represents the conceptual design of the testbed. A computer generates in phase and quadrature (IQ) waveform data and forwards them to a Software Defined Radio (SDR) device where the simulated signal is frequency modulated to AIS Class-A, Class-B or both channels in parallel. A signal attenuator is used to match testing signal power needs and to avoid saturation of the AIS receiver under test. The output of the receiver is then connected to the same computer to perform real-time comparison and data evaluation. During a run of the testbed the binary data used to generate waveforms is written to file. The output of the receiver is packet-wise encapsulated AIVDM messages. A message-by-message comparison between the input bytes of the waveform generator and the output data of the receiver provides the test result data set for the performance evaluation. As the smallest AIS packet is 256 bits long, it is also the packet size for test runs used. The received data stream is in real-time compared with the generated data and the results are plotted as graphs while the test is running.

The SDR Device used for the test setup is the USRP2 containing the WBX daughterboard from Ettus Research with a maximum output power of $200 \mathrm{~mW}$. In certain cases where waveforms were short, the signal generator SMBV100A from Rhode\&Schwarz was used instead.

To use an optimal and linear working range for the SDR, several measurements were made of the maximum output power of the SDR at different analog and digital gains. The measurements were made during the generation of an AIS test signal with a Rhode\&Schwarz ESU 26 EMI Test Receiver at a frequency of $162.025 \mathrm{MHz}$, by reading the maximal value in $\mathrm{dBm}$. The measurements in Fig.2 show that the gain is not

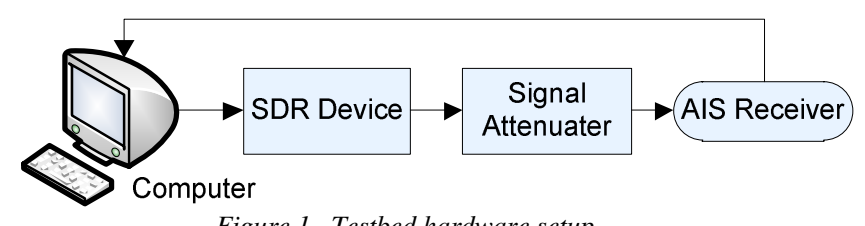

Figure 1. Testbed hardware setup 


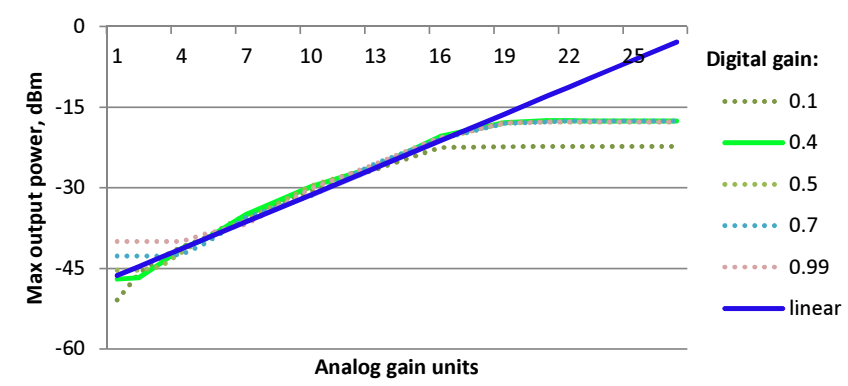

Figure 2. SDR output power for different gain setting

linear over the whole analog and digital gain setting range. Therefore, to have linear power increase steps within the SDRdriven test runs, a digital gain of 0.4 and an analog gain in the range of 5 to 21 units was used, providing power steering flexibility of up to $26 \mathrm{~dB}$. During the tests the signal attenuator was set to $90 \mathrm{~dB}$, thus providing a signal power range from $-130 \mathrm{dBm}$ to $-104 \mathrm{dBm}$.

Also, to ensure stable and precise output frequency, an external $10 \mathrm{MHz}$ rubidium reference clock was used to drive the SDR device.

Three different approaches of tests have been defined addressing the whole range of AIS receiver performance with a special emphasis on space applications:

- Basic tests on the physical layer characteristics with random AIS data

- Advance tests on the demodulation characteristics with random AIS data and

- High performance tests with simulated real-world dynamic data as seen by a satellite.

The test approaches are detailed in the following chapters, demonstrating their capabilities for SAT-AIS receiver testing.

\section{TESTS FOR CHARACTERISTIC PERFORMANCES OF PHYSICAL LAYER}

To check the performance of the physical layers of each AIS receiver, a set of different tests for single-message demodulation has been defined, addressing the receiver's sensitivity and bandwidth, as shown in Table 1. The AIS messages generated by the "AIS testbed" are data bursts for a single time slot and with random generated content, programmed in $\mathrm{C}++$ and $\mathrm{Py}-$ thon and based on a GNU-Radio DSP library.

TABLE 1. PHYSICAL LAYER TEST SCENARIOS

\begin{tabular}{lll} 
& \multicolumn{1}{c}{ Name } & \multicolumn{1}{c}{ Activity } \\
\hline III.1 & Receiver sensitivity & Stepwise turn up signal power. \\
\hline III.2 & $\begin{array}{l}\text { Receive channel band- } \\
\text { width }\end{array}$ & Shift signal in $-5 \mathrm{kHz} . .+5 \mathrm{kHz}$ frequency. \\
\hline III.3 & Doppler resistivity & Test III.2 with lean power level. \\
\hline III.4 & Disturbing signal & $\begin{array}{l}\text { While having one signal in center frequency, } \\
\text { shift second signal with Doppler. }\end{array}$
\end{tabular}

To get a good statistical basis, 1000 AIS messages were generated in each step and compared with the demodulated messages of the AIS receiver under test. For the tests previewed in this paper a commercial terrestrial AIS receiver was selected and, as an example, tested at the $162.025 \mathrm{MHz}$ center frequency. Typical examples of result plots for the sensitivity and frequency tests are shown below in Fig. 3 to 6 . The red line denotes messages received.

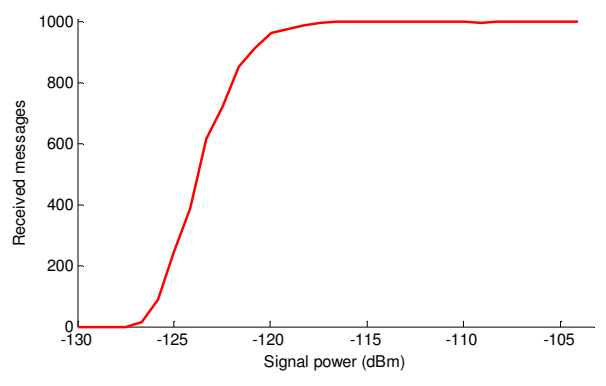

Figure 3. Result of sensitivity test, 1000 samples per step.

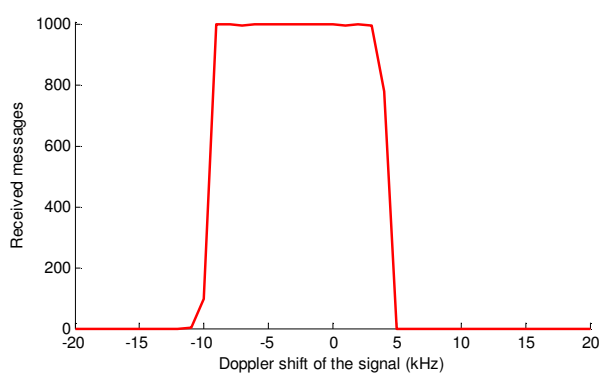

Figure 4. Result of frequency width test in -20 to $+20 \mathrm{kHz}$ range with $0.5 \mathrm{kHz}$ step at power level $-108 \mathrm{dBm}, 1000$ samples per step.

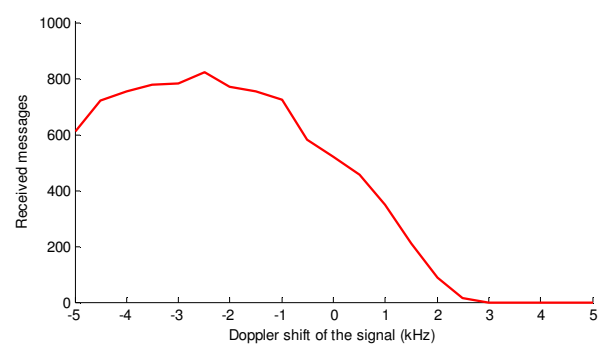

Figure 5. Result of frequency width test in the -5 to $+5 \mathrm{kHz}$ range with $0.5 \mathrm{kHz}$ step at power level of $-122 \mathrm{dBm}, 1000$ samples per step.

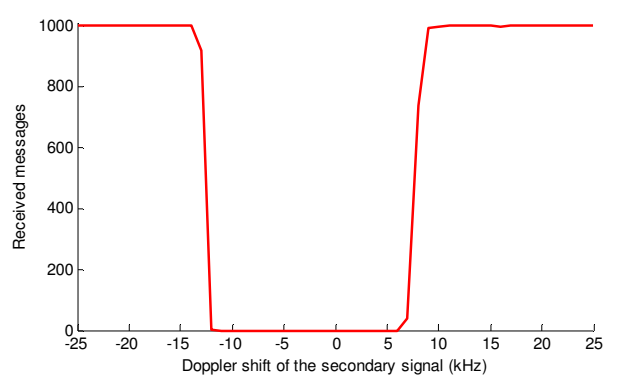

Figure 6. Result sample of disturbing signal test. One signal is standing at middle frequency while the second is being shifted in the -25 to $+25 \mathrm{kHz}$ range, both at power level $-108 \mathrm{dBm}$, with $0.5 \mathrm{kHz}$ step, 1000 samples per step. 
Interpreting the figures the following conclusions on receiver performance can be made.

III.1) Sensitivity. The receiver is able to start the demodulation of AIS signals at $-127 \mathrm{dBm}$ and performs robust demodulation from $-117 \mathrm{dBm}$ onwards. Thermal noise level was $-130 \mathrm{dBm}$.

III.2) Channel bandwidth. At good power level $-108 \mathrm{dBm}$, the receiver is able to demodulate signals with frequency shifts from $-10 \mathrm{kHz}$ to $+5 \mathrm{kHz}$. Fig. 4 clearly shows that the center frequency of the used AIS receiver is misaligned.

III.3) Doppler resistivity at lean signal power. At a level of $-122 \mathrm{dBm}$ the receiver is able to demodulate maximum $80 \%$ of the messages within the range of $\pm 5 \mathrm{kHz}$ from its center frequency. The demodulation probability diminishes with frequency shift from center. Here it is obvious that the receiver's center frequency is shifted approximately $2.5 \mathrm{kHz}$ down for the particular channel, here $162.025 \mathrm{MHz}$.

III.4) Disturbing signal. At Doppler shifts ranging from -12 to $+7 \mathrm{kHz}$ the second parallel signal is disturbing the central one at the middle frequency of $162.025 \mathrm{MHz}$. This means, that the receiver has no resistance against another signal of the same power, in the whole possible $\pm 4 \mathrm{kHz}$ [4] Doppler range of a LEO satellite-to-ship communication.

The above results show that by means of a testbed run, basic characteristics of the AIS receiver's physical layer can be determined which are considered important for any receiver's operation.

\section{SPACE-AIS SPECIFIC DEMODULATION ALGORITHM TESTS}

Apart from the basic characteristic tests as described in the previous chapter, there are additional specific test cases which allow evaluation of demodulation algorithm performances for some specific SAT-AIS cases. Table 2 outlines test definitions which are implemented in MATLAB and executed by the signal generator.

IV.1) Single bit error. Flipping one bit in the data or CRC fields would lead a standard receiver to discard the whole message. This dedicated test shows if a single-bit error recovery is implemented. If the wrong bit occurs within the 168 bits of the data field, a check through all 168 alternative checksum cases could lead to recovery. And in case of an error in the CRC field, a single bit flip there would have to match the present data CRC. The tested receiver did not have this option.

TABLE 2. ALGORITHM-SPECIFIC TEST SCENARIOS

\begin{tabular}{lll} 
& Name & Activity \\
\hline IV.1 & Single bit error & Generate signal with one wrong bit \\
\hline IV.2 & $\begin{array}{l}\text { Path delay over- } \\
\text { laps }\end{array}$ & $\begin{array}{l}\text { Cut away the } 0 . .32 \text { and 224..256 bits from single- } \\
\text { slot packet. }\end{array}$ \\
\hline IV.3 & $\begin{array}{l}\text { Double-Doppler } \\
\text { shifts }\end{array}$ & $\begin{array}{l}\text { Overlap two messages, each Doppler shifted to } \\
\text { different sides from center. Stepwise reduce the } \\
\text { frequency difference to 4kHz. }\end{array}$ \\
\hline IV.4 & Full overlap case & $\begin{array}{l}\text { Overlap two signals of weights 1:0.3. Stepwise } \\
\text { increase magnitude of the second signal. }\end{array}$ \\
\hline IV.5 & $\begin{array}{l}\text { Multi-message } \\
\text { case }\end{array}$ & $\begin{array}{l}\text { Overlap multiple messages, vary power ratios, } \\
\text { frequency and phase shifts. }\end{array}$
\end{tabular}

IV.2) Path delay overlaps. SAT-AIS receivers face the problem of message overlaps due to different propagation delays, leading to truncated messages. For instance, the end flag may be overlapped by start bits of a subsequent AIVDM message. Terrestrial AIS receivers would interpret overlapped bits and/or a missing end flag of the subsequent message incorrectly, leading to unidentified and eventually discarded packets. However, smart algorithms implemented in the SAT-AIS receiver might identify message endings with matching CRC, by counting from the packet starting flag for 1-slot message bits, including $\mathrm{CRC}$ and bit stuffing. A similar approach may be used to validate missing start-bits: identify message by counting backwards from packet end flag. Since start and end flags of an AIS packet are equal, the forward and back checks would have to be done in parallel.

Tests of the example AIS receiver showed that an 8-bit ramp-up time cut was not significant, giving still $100 \%$ reception, whereas further truncations from the 9 th to 18 th bit led to a lower identified message rate from $100 \%$ down to $0 \%$, due to the inability to attain phase lock. Also, as expected, no demodulation was possible if parts of the start or finishing flag were missing. To overcome partly or missing training sequence problem, the proposed algorithm in [6] would help to derive frequency and phase lock, by using the baseband signal of the data bits.

IV.3) Double Doppler shifts. this test gives an indication if adaptive narrow-band filters are working properly. Since the transmission bandwidth bit-time product (BT) is 0.4 for AIS messages, it has by definition a $-3 \mathrm{~dB}$ signal strength at a $3.84 \mathrm{kHz}$ frequency offset from the central frequency. In case of two AIS signals separated by $4 \mathrm{kHz}$ due to opposite $2 \mathrm{kHz}$ Doppler shifts - which is typical for LEO satellites - a demodulation should be possible for 2 signals having the same power level when highly accurate frequency filters are present. This test thus gives important information about the receiver's capability to simultaneously demodulate multiple parallel signals with significant Doppler shifts. An example of a receiver having this implemented is given in [5].

IV.4) Full overlap cases. This test could be considered one of the most significant and also the hardest for SAT-AIS receiver designs to pass successfully: namely to demodulate overlapped messages. There are several subtest scenarios of interest, among them the following two of the most interest: the test of Demod-Remod and the test of Joint MLSE (JMLSE) multi-message demodulation algorithm presence, iteration depth and performance levels.

For the Demod-Remod subscenario, at least two signals with power ratio $0 \mathrm{~dB}$ vs. $-9 \mathrm{~dB}$ are generated and the power level of the second signal is stepwise increased to $-3 \mathrm{~dB}$. The result shows the receiver capability to demodulate consecutively added signals with significant gain differences. The scenario is specially designed for identification and performance evaluation of the Demod-Remod or similar overlapped signal demodulation methods within the receiver. In the case overlapping signal powers differ by at least $3 \mathrm{~dB}$, the signals can be consecutively subtracted one from another, with demodulation starting from the strongest one. An appropriate application of 
this algorithm with using precise frequency evaluation for remodulation is described in [4].

The JMLSE subscenario simulation is very similar to the Demod-Remod approach, but with the difference that the overlapping signal may be as powerful as the main signal while phases stay shifted. The JMLSE method is computationally very intensive [7], but promises an improvement in SAT-AIS receiver demodulation capabilities.

The tests identified above are considered to be the main performance evaluation criteria for assessing demodulation algorithm performances of SAT-AIS receivers. As the receiver under test was unable to deal with any of the space-specific problems, results are not discussed here in detail.

\section{TESTS WITH REALISTIC SIMULATED DATA}

The third test approach, also implemented in the DLR AIS test bed, contains a vessel scenario as observed by a LEO satellite, to eventually incorporate the SOTDMA protocol as exchanged between individual ships. Fig. 7 shows a concise design diagram for the entire simulation process.

Ship Generator: an input AIS file containing a ship database is passed on for system initialization, reflecting a ship traffic snapshot at a given time. These data define the initial positions of the vessels and are linearly extrapolated to simulate the ship movement. This gives a good approximation of the positions and the respective AIS message content during a pass of a LEO satellite, which is typically around 10 minutes. Two Line Element data is defined to describe the satellite's orbit and to calculate dedicated orbit positions, angles, distances and velocities to any simulated ship. The modelled data set is written in an "AIS message-time frame".

Signal Modulator: the simulated AIS data set is converted into IQ data incorporating timing delays and signal attenuations due to the geometric distance (between satellite and ship), the antenna angle and Doppler shifts. For cases when signals for satellite Field Of View (FOV) are colliding, the corresponding overlaps are made in the IQ waveforms.
This builds up a very realistic ship traffic scenario as seen by a SAT-AIS receiver onboard a satellite. The IQ data are then frequency modulated by means of a SDR or a signal generator and input into the SAT-AIS receiver under test.

Following the ship traffic density classification as presented in [2], Table 3 summarizes considered test scenarios for different situation models, reflecting realistic environment tests.

Fig. 8 represents a 30 second simulation of an artificial satellite pass over the North Sea, covering 1218 modelled ships. Source data are taken from an aeronautical campaign acquired during a flight over the German Bight in the frame of the DLR AIS research activities. Each of the circles displayed correspond to a ship and contain a series of time-position points corresponding to AIS transmissions by that certain ship.

This test with realistic AIS messages has significant advantages compared to the two previously described test approaches performed with random content data. For instance, it can serve as a basis for smart post-processing tests by using information from properly demodulated messages to correct some wrong bits of incoming messages of the same ship or, more advanced, for next message arrival time prediction or data set compression algorithm evaluation.

In the current simulator version, voyage report messages of type 1, 2 and 3 are implemented, which provide a sound basis for test runs.

The 30 second long test was prepared for the simulated situation, using signal generator and receiver. The following model settings were used:

- minimal visibility angle ship-to-satellite: 5 degrees;

- $\quad$ ship transmission power: random between 11 and 12 Watts;

- $\quad$ satellite antenna gain: $\operatorname{simulated~gain~} 3 \cdot \sin (x)$, where $x$ is the nadir angle of satellite-to-ship view, and the $3 \mathrm{~dB}$ is the directional gain of the antenna;

- $\quad$ orbit altitude: $720 \mathrm{~km}$.

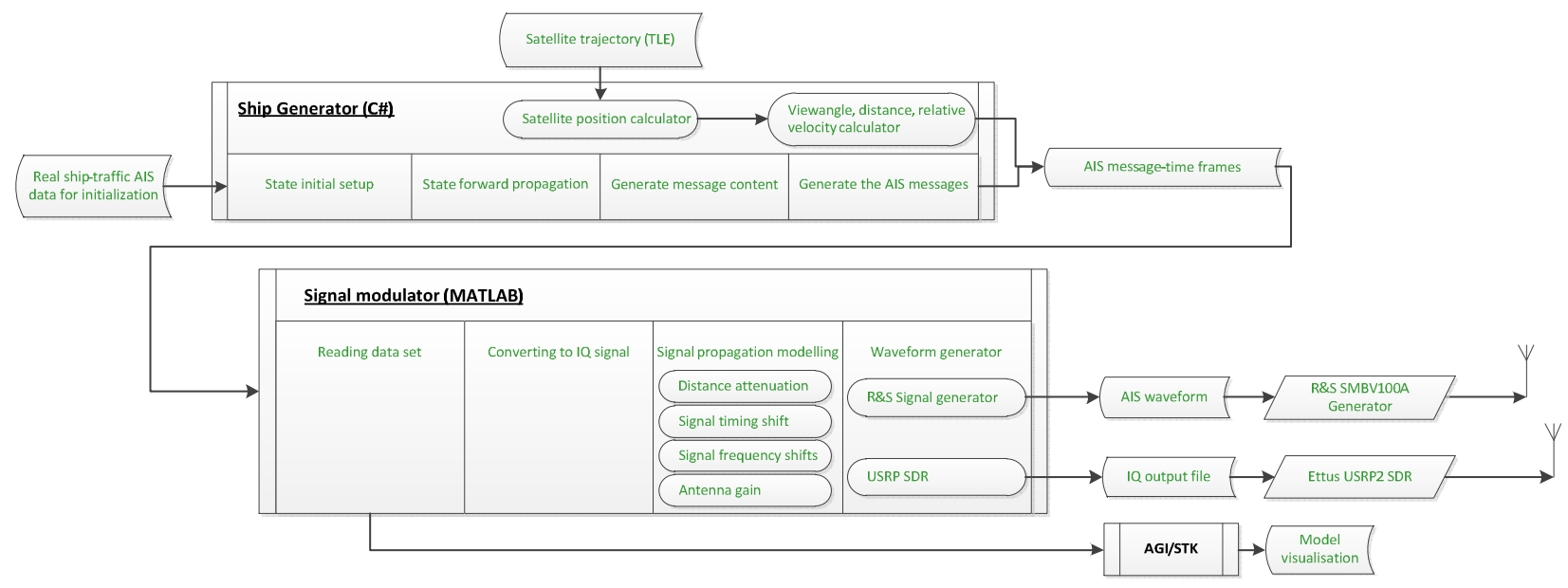

Figure 7. Data flow within propagator software for real orbit like RF signal generation 
TABLE 3. TESTING SCENARIOS BASED ON REAL WORLD DATA

\begin{tabular}{|c|c|c|}
\hline & Name & Activity \\
\hline V.1 & Low density traffic & \multirow{4}{*}{$\begin{array}{l}\text { Run with evenly spaced random data } \\
\text { and with real data sample. Vary satel- } \\
\text { lite orbit, antenna parameters. }\end{array}$} \\
\hline V.2 & Medium density traffic & \\
\hline V.3 & High density real traffic & \\
\hline V.4 & Mixed & \\
\hline
\end{tabular}

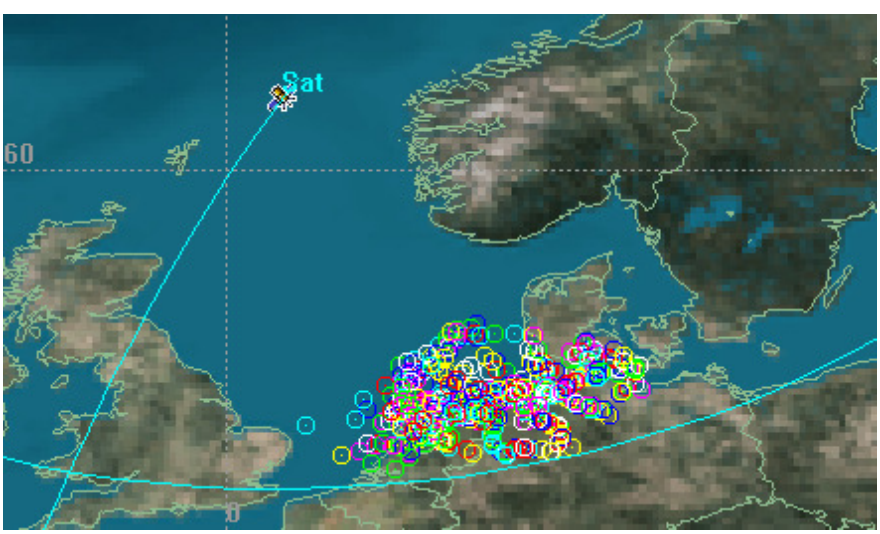

Figure 8. Data flow within propagator for in-orbit alike RF signal generation, view generated with AGI/STK

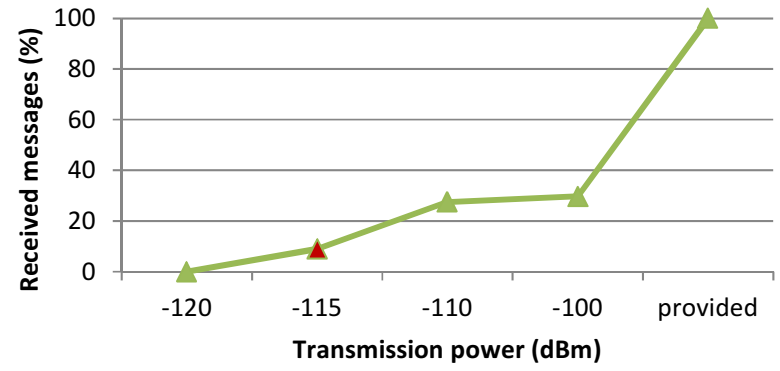

Figure 9. Results of 30 seconds realistic simulated environment test at different power levels. The set "provided" correspond to initial number of messages modulated. The set 115 corresponds to the real-world environment.

Preliminary test results are shown in Fig. 9. The signal generator's transmission power level is set to $-115 \mathrm{~dB}$ for the realworld situation. For a comparison, some neighboring transmission powers are also shown in the graph. One can observe that increasing signal power increases the number of received messages, showing that signal strength or antenna booth could help receiption until overlapping takes place. In this particular simulation set-up (orbit, antenna, ship traffic and AIS receiver) only $9 \%$ of all messages were found to be demodulated. This may give a good hint for the reduced demodulation capabilities of SAT-AIS in high traffic areas.
Detailed investigation on how this simulation model is built and more of its application approaches, including analysis on how demodulation performance is influenced by each of the signal disturbing factors separately, will be discussed in forthcoming papers.

\section{OUTLOOK TO APPLICABILITY OF THE TESTS}

In the frame of the DLR AISat satellite project, a test bed for SAT-AIS receivers has been developed, applying different test scenarios with different AIS data qualities to provide a means to evaluate and analyze their performance figures independently. This has been achieved by a combination of AIS data generation and RF signal characteristics simulation.

As a result it can be stated that it is generally possible to derive the characteristics of the demodulation and smart processing capabilities for a receiver by using a test bed. The three discussed test approaches, incorporating the twelve different tests, reflected in the three tables of this paper are considered to provide a promising approach to assess any SATAIS hardware performance.

\section{REFERENCES}

[1] "Technical characteristics for an automatic identification system using time-division multiple access in the VHF maritime mobile band," recommendation ITU-R M.1371-4.

[2] M.A. Cervera, A. Ginesi, "On the Performance Analysis of a Satellitebased AIS System", 10th International Workshop on Signal Processing for Space Communications (SPSC), 2008.

[3] F. te Hennepe, R. Rinaldo, A. Ginesi, C. Tobehn, M. Wieser, Ø. Olsen, $\emptyset$. Helleren, R. Challamel, F. Storesund, "Space-Based Detection of AIS Signals," 5th Advanced satellite multimedia systems conference (ASMA) and the 11th signal processing for space communications workshop (SPSC), 2010

[4] P. Burzigotti, A. Ginesi, G. Colavolpe, "Advanced receiver design for satellite-based AIS signal detection," 5th Advanced satellite multimedia systems conference (ASMA) and the 11th signal processing for space communications workshop (SPSC), 2010

[5] Jesper A.Larsen, Hans Peter Mortensen, Jens D.Nielsen, "An SDR based AIS Receiver for Satellites," Recent Advances in Space Technologies (RAST), 2011 5th International Conference, 2011.

[6] M.J. Gallardo, U.A. Sorger, "Coherent receiver for AIS satellite detection," 4th International Symposium on Communications, Control and Signal Processing (ISCCSP), 2010.

[7] P.A. Murphy, G.E. Ford, "Co-channel demodulation for continuousphase modulated signals," Conference Record of the Twenty-Ninth Asilomar Conference on Signals, Systems and Computers, 1995, 330 334 vol.1.

[8] A. Scorzolini, V. De Perini, E. Razzano, G. Colavolpe, S. Mendes, P. Fiori, A. Sorbo, "European Enhanced Space-based AIS System Study," 5th Advanced satellite multimedia systems conference (ASMA) and the 11th signal processing for space communications workshop (SPSC), 2010. 